\title{
A Mathematical Reformulation of Derrida's REM and GREM
}

\author{
David Ruelle \\ I.H.E.S., 35, Route de Chartres, F-91440 Bures-sur-Yvette, France
}

\begin{abstract}
The large system limit of the Random Energy Model (REM) and generalized Random Energy Model (GREM) of Derrida is investigated, and found to be universal. This permits systematic calculations of relevance in particular to Parisi's solution of the Sherrington-Kirkpatrick spin-glass model.
\end{abstract}

\section{Introduction}

B. Derrida has recently introduced two statistical models called respectively Random Energy Model (REM) [2,3] and Generalized Random Energy Model (GREM) $[4,5]$. These models are particularly interesting because they describe the thermodynamic behavior of the Sherrington-Kirkpatrick (SK) model $[14,8]$ expected on the basis of Parisi's Ansatz. For a discussion of the SK model in the light of Parisi's Ansatz [12,13], we refer to Mézard et al. [9,11], and references quoted there. For the connection with the REM and GREM, see Mézard, Parisi and Virasoro [10], Derrida and Toulouse [6] and, most clearly, de Dominicis and Hilhorst [7].

In Derrida's formulation of the REM and GREM, certain limits are implicit $(N \rightarrow \infty$, and for the GREM, number of levels of the hierarchy $\rightarrow \infty)$. The purpose of the present paper is to give a mathematical reformulation where the appropriate limits have already been taken. Our approach has the advantage of showing that no hidden difficulties lurk behind these limits. It also permits an easier discussion of certain problems, as we shall see below.

We shall proceed dogmatically by defining certain spaces and probability measures. The connection with Derrida's definitions should then be rather clear, and is discussed only briefly.

\section{Poisson Distributions}

The usual Poisson distribution describes (infinite) configurations of points on the line $\mathbb{R}$, with given density $\varphi$, and such that any two disjoint intervals of $\mathbb{R}$ behave independently. This setup can be variously generalized, we shall use an extension where $\mathbb{R}$ is replaced by a nonempty open set $\mathcal{O} \subset \mathbb{R}^{v}$, and the density by a continuous function $\varphi \geqslant 0$ on $\mathcal{O}$. A configuration $X$ of points in $\mathcal{O}$ will be represented by an 
occupancy function $X: \mathcal{O} \rightarrow \mathbb{N}$ (the natural integers $0,1,2, \ldots)$ such that $\sum_{\xi \in K} X(\xi)<$ $+\infty$ whenever $K \subset \mathcal{O}$, and $K$ is compact. ${ }^{1}$ This means that points of the configuration can accumulate only on the boundary of $\mathcal{O}$ or at $\infty$; multiple occupancy is allowed but will turn out to have zero probability.

The space $\mathscr{X}(\mathcal{O})$ of allowed configurations in $\mathcal{O}$ has a natural topology corresponding to convergence away from the boundary of $\mathcal{O}$ and $\infty$ (see Appendix 1 for a precise definition). To the topology of $\mathscr{X}(\mathcal{O})$ is attached a Borel structure, which can be used to define Borel probability measures on $\mathscr{X}(\mathcal{O})$.

Let now $B$ be a relatively compact Borel subset of $\mathcal{O}$ (i.e. $B$ is a bounded Borel subset of $\mathbb{R}^{v}$, and closure $\left.B \subset \mathcal{O}\right)$. We denote by $\mathscr{X}(B)\left(\right.$ respectively $\mathscr{X}^{m}(B)$ ) the space of functions $Y: B \rightarrow \mathbb{N}$ such that $\sum_{\xi \in B} Y(\xi)<+\infty$ (respectively $\sum_{\xi \in B} Y(\xi)=m$ ). Clearly $\mathscr{X}(B)$ is the disjoint union of the $\mathscr{X}^{m}(B)$ :

$$
\mathscr{X}(B)=\sum_{m \in \mathbb{N}} \mathscr{X}^{m}(B)
$$

There is a natural Borel structure on each $\mathscr{X}^{m}(B)$, and therefore also on $\mathscr{X}(B)$. The map $\pi_{B}: \mathscr{X}(\mathcal{O}) \rightarrow \mathscr{X}(B)$ which sends $X \in \mathscr{X}(\mathcal{O})$ to its restriction $\pi_{B} X=X \mid B$ is Borel $^{2}$. We shall also need the Borel map $Y: B^{m} \rightarrow \mathscr{X}^{m}(B)$ such that

$$
\left(Y\left(\xi_{1}, \ldots, \xi_{m}\right)\right)(\eta)=\left(\text { number of indices } i \text { such that } \xi_{i}=\eta\right) \text {. }
$$

Finally, if $B_{1}, B_{2}$ are relatively compact Borel subsets of $\mathcal{O}$, and $B_{1} \cap B_{2}=\phi$, we can make the identification

$$
\mathscr{X}\left(B_{1} \cup B_{2}\right)=\mathscr{X}\left(B_{1}\right) \times \mathscr{X}\left(B_{2}\right)
$$

where $Y: B_{1} \cup B_{2} \rightarrow \mathbb{N}$ corresponds to $\left(Y \mid B_{1}\right) \times\left(Y \mid B_{2}\right)$.

We are now equipped to define Poisson distributions. First we consider a relatively compact Borel set $B \subset \mathcal{O}$, and a Lebesgue integrable function $\varphi \geqq 0$ on $B$. The Poisson distribution corresponding to $B$ and $\varphi$ is the probability measure $m_{\varphi}^{B}$ defined on $\mathscr{X}(B)$ by

$$
m_{\varphi}^{B}(A)=\int m_{\varphi}^{B}(d X) A(X)=\left(\Xi_{\varphi}^{B}\right)^{-1} \sum_{m=0}^{\infty} \frac{1}{m !} \int_{B} \varphi\left(\xi_{1}\right) d \xi_{1} \cdots \int_{B} \varphi\left(\xi_{m}\right) d \xi_{m} A\left(Y\left(\xi_{1}, \ldots, \xi_{m}\right)\right)
$$

when $A$ is a bounded Borel function on $\mathscr{X}(B)$, and we have written

$$
\Xi_{\varphi}^{B}=\exp \int_{B} \varphi(\xi) d \xi
$$

\footnotetext{
1 The reader may choose to go rather lightly over the details given below, and ignore completely the Appendix. Physically, the Poisson distribution $m_{\varphi}$ corresponds to the distribution of positions of particles in a free gas at temperature 1, contained in the (usually infinite) region $\mathcal{O}$, the particles of the gas being subjected to an external potential $-\log \varphi$

2 Note that for compact $K \subset \mathcal{O}, \mathscr{X}(K)$ has a natural locally compact topology, and that $\pi_{K}: \mathscr{X}(O) \rightarrow \mathscr{X}(K)$, which is Borel, is not continuous
} 
It is readily checked that, corresponding to (1.1), the following formula holds:

$$
m_{\varphi}^{B_{1} \cup B_{2}}=m_{\varphi \mid B_{1}}^{B_{1}} \times m_{\varphi \mid B_{2}}^{B_{2}} .
$$

Let now $\varphi \geqq 0$ be a locally integrable function on $\mathcal{O}$ (i.e. such that $\int_{K} \varphi(\xi) d \xi<+\infty$ for every compact set $K \subset \mathcal{O}$ ). We define the Poisson distribution corresponding to $\varphi$ as the probability measure $m_{\varphi}$ on $\mathscr{X}(\mathcal{O})$ such that $\pi_{B} m_{\varphi}=m_{\varphi \mid B}^{B}$ for every relatively compact Borel set $B \subset \mathcal{O}$. If $\mathcal{O}$ is written as a countable union of disjoint relatively compact Borel subsets $B_{\alpha}$, one can make the identification

$$
m_{\varphi}=\Pi_{\alpha} m_{\varphi \mid B_{\alpha}}^{B_{\alpha}} .
$$

Using (1.3) one can see that this definition is unique.

Consider now another open set $\mathcal{O}^{*} \subset \mathbb{R}^{v}$, and let $f: \mathcal{O} \rightarrow \mathcal{O}^{*}$ be a diffeomorphism (i.e. $f$ has a unique inverse $f^{-1}: \mathcal{O}^{*} \rightarrow \mathcal{O}$, and both $f, f^{-1}$ are differentiable). It is not hard to see that the image by $f$ of the Poisson distribution $m_{\varphi}$ on $\mathscr{X}(\mathcal{O})$ is a Poisson distribution $m_{\varphi^{*}}$ on $\mathscr{X}\left(\mathcal{O}^{*}\right)$ :

$$
f m_{\varphi}=m_{\varphi^{*}}, \quad \varphi^{*}(\eta)=\left|J\left(f^{-1} \eta\right)\right|^{-1} \varphi\left(f^{-1} \eta\right),
$$

where $J$ denotes the Jacobian determinant:

$$
J(\xi)=\operatorname{det}\left(\partial f_{i} / \partial \xi_{j}\right)
$$

\section{The Random Energy Model (REM)}

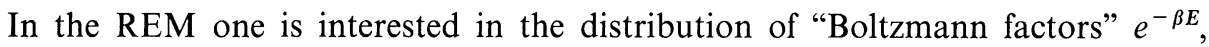
where the $E$ have a Poisson distribution, and its density $\varphi$ on $\mathbb{R}$ is exponential ${ }^{3}$ : $\varphi(\xi)=\rho e^{\rho \xi}$. We are in the situation of the preceding section, with $\mathcal{O}=\mathbb{R}, \mathcal{O}^{*}=$ $\mathbb{R}_{>}=\{\eta \in \mathbb{R}: \eta>0\}$ and $f(\xi)=e^{-\beta \xi}$, with $0<\rho<\beta$. Therefore the "Boltzmann factors" have a distribution with density $\varphi^{*}$ on $\mathbb{R}_{>}$such that

$$
\varphi^{*}(\eta)=\left|-\beta \exp -\beta\left(-\frac{1}{\beta} \log \eta\right)\right|^{-1} \rho \exp \left[\rho\left(-\frac{1}{\beta} \log \eta\right)\right]=\beta^{-1} \eta^{-1} \rho \eta^{-\rho / \beta}
$$

(see Eq. (1.4)). Defining $x=\rho / \beta \in(0,1)$, we have thus $\varphi^{*}(\xi)=x \xi^{-x-1}$. Since $\varphi^{*}$ depends on $\rho, \beta$ only through $x$, we shall write $\varphi^{*}=\varphi_{x}, m_{\varphi^{*}}=m_{x}$.

Note that $m_{x}$ is a measure on objects $X$ which are unordered sequences $X=\left\{\xi_{\alpha}\right\}$ of points of $\mathbb{R}_{>}$, such that $\xi_{\alpha} \rightarrow 0$ with probability 1 . Write $|X|=\Sigma \xi_{\alpha}$. It is easy to see that $|X|<\infty$ for $m_{x}-$ almost all $X$, but

$$
\int|X| m_{x}(d X)=\int_{0}^{\infty} x \xi^{-x-1} \xi d \xi=\infty
$$

(this is readily obtained from (1.2) by a limit of the type used in the proof of the next proposition). The map $X \rightarrow X /|X|$ sends $m_{x}$ to a measure $\tilde{m}_{x}$ on (unordered)

3 In Derrida's original formulation [2,3], $\varphi$ is taken to be Gaussian, so that $\int \varphi(\xi) d \xi$ is finite; a limit $N$ $\rightarrow \infty$ is subsequently performed. In the equivalent treatment of Mézard, Parisi and Virasoro [10], $\varphi$ is exponential, but with a cut off so that $\int \varphi(\xi) d \xi$ is again finite; here a limit $M, f_{c} \rightarrow \infty$ has to be performed. The use of Poisson distributions avoids these limits 
sequences $\tilde{X}=\left\{\tilde{\xi}_{\alpha}\right\}$ with $\Sigma_{\alpha} \xi_{\alpha}=1$. Note that $\tilde{m}_{x}$ is not a Poisson distribution. The study of the probability measure $\tilde{m}_{x}$ constitutes a good part of the literature on the REM and of the treatment of the SK model based on Parisi's Ansatz. The proof of the proposition below shows that it is particularly easy to discuss $\tilde{m}_{x}$ using the fact that $m_{x}$ is a Poisson distribution.

2.1. Proposition. Define a density $\rho_{x}$ associated with $\tilde{m}_{x}$ such that

Then

$$
\rho_{x}(p)=\int \tilde{m}_{x}(d \tilde{X}) \sum_{\alpha} \delta\left(p-\tilde{\xi}_{\alpha}\right)
$$

$$
\rho_{x}(p)=\frac{(1-p)^{x-1} p^{-x-1}}{\Gamma(x) \Gamma(1-x)}
$$

It is easily verified that

$$
\rho_{x}(p)=\int m_{x}(d X) \sum_{\alpha} \delta\left(p-\xi_{\alpha} /|X|\right)=\lim _{\varepsilon \rightarrow 0} \int m_{x}^{\varepsilon}(d X) \sum_{\alpha} \delta\left(p-\xi_{\alpha} /|X|\right),
$$

where $m_{x}^{\varepsilon}$ is the Poisson distribution associated with the density $\varphi_{x}^{\varepsilon}$ such that

$$
\varphi_{x}^{\varepsilon}(\xi)=\left\{\begin{array}{cc}
0 & \text { if } \xi<\varepsilon \\
x \xi^{-x-1} & \text { if } \xi>\varepsilon
\end{array} .\right.
$$

With the obvious definition of $\Xi$ we have

$$
\begin{aligned}
\int m_{x}^{\varepsilon}(d X) \sum_{\alpha} \delta\left(p-\xi_{\alpha} /|X|\right)= & \Xi^{-1} \sum_{m=0}^{\infty} \frac{1}{m !} \int \varphi_{x}^{\varepsilon}\left(\xi_{1}\right) d \xi_{1} \cdots \int \varphi_{x}^{\varepsilon}\left(\xi_{m}\right) d \xi_{m} \\
& \cdot \sum_{k=1}^{m} \delta\left(p-\xi_{k} /\left(\xi_{1}+\cdots+\xi_{m}\right)\right) \\
= & \Xi^{-1} \sum_{m=0}^{\infty} \frac{1}{m !} m \int \varphi_{x}^{\varepsilon}\left(\xi_{1}\right) d \xi_{1} \cdots \int \varphi_{x}^{\varepsilon}\left(\xi_{m-1}\right) d \xi_{m-1} \int \varphi_{x}^{\varepsilon}(\xi) d \xi \\
& \cdot \delta\left(p-\xi /\left(\xi_{1}+\cdots+\xi_{m-1}+\xi\right)\right) \\
= & \Xi^{-1} \sum_{n=0}^{\infty} \frac{1}{n !} \int \varphi_{x}^{\varepsilon}\left(\xi_{1}\right) d \xi_{1} \cdots \int \varphi_{x}^{\varepsilon}\left(\xi_{n}\right) d \xi_{n} \\
& \cdot \int \varphi_{x}^{\varepsilon}(\xi) d \xi\left(\xi_{1}+\cdots+\xi_{n}+\xi\right) \frac{1}{1-p} \delta\left(\left(\xi_{1}+\cdots+\xi_{n}\right) \frac{p}{1-p}-\xi\right) \\
= & \int m_{x}^{\varepsilon}(d X) \varphi_{x}^{\varepsilon}\left(|X| \frac{p}{1-p}\right)\left(|X|+|X| \frac{p}{1-p}\right) \frac{1}{1-p} \\
= & \int m_{x}^{\varepsilon}(d X) \frac{|X|}{(1-p)^{2}} \varphi_{x}^{\varepsilon}\left(|X| \frac{p}{1-p}\right) .
\end{aligned}
$$

Letting $\varepsilon \rightarrow 0$, we obtain

$$
\begin{aligned}
\rho_{x}(p) & =\int m_{x}(d X) \frac{|X|}{(1-p)^{2}} \varphi_{x}\left(|X| \frac{p}{1-p}\right)=\frac{1}{(1-p)^{2}}\left(\frac{p}{1-p}\right)^{-x-1} x \int m_{x}(d X)|X|^{-x} \\
& =C^{-1}(1-p)^{x-1} p^{-x-1}
\end{aligned}
$$


Since $\int_{0}^{1} \rho_{x}(p) p d p=1$, we have

$$
C=\int_{0}^{1} p^{-x-1}(1-p)^{x-1} d p=\Gamma(x) \Gamma(1-x),
$$

and the proposition follows.

2.2. Corollary. (a) We have

In particular,

$$
\int_{0}^{1} \rho_{x}(p) p^{k} d p=\frac{\Gamma(k-n)}{\Gamma(1-x) \Gamma(k)}
$$

$$
\int_{0}^{1} \rho_{x}(p) p^{2} d p=1-x
$$

(b) From (2.1) and (2.2) we obtain also

$$
x \int m_{x}(d X)|X|^{-x}=\frac{1}{\Gamma(x) \Gamma(1-x)}=\frac{\sin \pi x}{\pi} .
$$

(c) With a proof similar to that of the Proposition, we have, for Res $<x$,

$$
\left[\int m_{x}(d x)|X|^{s}\right]^{-1} \int m_{x}(d X)|X|^{s} \sum_{\alpha} \delta\left(p-\frac{\xi_{\alpha}}{|X|}\right)=\frac{\Gamma(1-s)}{\Gamma(1-x) \Gamma(x-s)} p^{-x-1}(1-p)^{x-s-1}
$$

and

$$
\int m_{x}(d X)|X|^{s}=\frac{\Gamma(1-x) \Gamma(x-s)}{\Gamma(1-s)} x \int m_{x}(d X)|X|^{s-x} .
$$

(d) As a consequence of (b) and (c) we have also

$$
x \int m_{x}(d X)|X|^{-k x}=\frac{\Gamma(k)}{\Gamma(k x)(\Gamma(1-x))^{k}} .
$$

2.3. Proposition. Let $X \subset \mathbb{R}_{>}$be distributed according to $m_{x}(d X)$ (Poisson distribution with density $\varphi_{x}$ ). If $a>0$, then aX has a Poisson distribution with density $a^{x} \varphi_{x}$.

As discussed at the end of Sect.1, $a X$ has Poisson distribution with density $\varphi^{*}$ given by (1.4):

$$
\varphi^{*}(\eta)=a^{-1} \varphi_{x}\left(a^{-1} \eta\right)=a^{-1} x\left(a^{-1} \eta\right)^{-x-1}=a^{-1+x+1} x \eta^{-x-1}=a^{x} \varphi_{x}(\eta) .
$$

\section{The Generalized Random Energy Model (GREM)}

In this section, we shall describe the GREM as a probability measure $\tilde{m}_{n}\left(d \tilde{X}^{(n)}\right)$ on finite probability cascades $\tilde{X}^{(n)}$. This corresponds to the situation originally discussed by Derrida; in the next section we shall replace finite by continuous cascades.

We choose an integer $n \geqq 1$ and consider unordered families $\left(\xi_{\alpha_{1} \ldots \alpha_{n}}\right)$ with the following properties

(a) $\xi_{\alpha_{1} \ldots \alpha_{n}}>0$, 
(b) each index $\alpha_{1}, \ldots, \alpha_{n}$ runs over a countable infinite set, for which we may for instance take the set $\mathbb{N}$ of natural integers,

(c) $\xi_{\alpha_{1} \ldots \alpha_{n}} \rightarrow 0$ when $\alpha_{n} \rightarrow \infty$,

(d) the family $\left(\xi_{\alpha_{1} \ldots \alpha_{n}}\right)$ is unordered in the sense that we allow all permutations $\pi:\left(\alpha_{1}, \ldots, \alpha_{n}\right) \rightarrow\left(\alpha_{1}^{\prime}, \ldots, \alpha_{n}^{\prime}\right)$ such that if $\alpha_{1}=\beta_{1}, \ldots, \alpha_{j-1}=\beta_{j-1} \alpha_{j} \neq \beta_{j}$, then $\alpha_{1}^{\prime}=\beta_{1}^{\prime}, \ldots, \alpha_{j-1}^{\prime}=\beta_{j-1}^{\prime}, \alpha_{j}^{\prime} \neq \beta_{j}^{\prime}$ [in other words, we allow permutations which preserve the tree structure of the index set corresponding to the ultrametric distance $d\left(\left(\alpha_{1}, \ldots, \alpha_{n}\right),\left(\beta_{1}, \ldots, \beta_{n}\right)\right)=\exp \left(-\min \left\{j: \alpha_{j} \neq \beta_{j}\right\}\right)$.

Note that for $n=1$, the unordered families just described reduce to the unordered sequences of Sect. 2 .

If $\pi:\left(\alpha_{1}, \ldots, \alpha_{n}\right) \rightarrow\left(\alpha_{1}^{\prime}, \ldots, \alpha_{n}^{\prime}\right)$ is an allowed permutation then, in view of (d), for each $j$ (from 1 to $n$ ) there is a uniquely defined permutation $\left(\alpha_{1}, \ldots, \alpha_{j}\right) \rightarrow\left(\alpha_{1}^{\prime}, \ldots \alpha_{j}^{\prime}\right)$, which we shall again denote by $\pi$.

We may now consider cascades,

$$
X^{(n)}=\left(\xi_{\alpha_{1}}^{1}, \xi_{\alpha_{1} \alpha_{2}}^{2}, \ldots, \xi_{\alpha_{1} \ldots \alpha_{n}}^{n}\right),
$$

where the $\left(\xi_{\alpha_{1} \ldots \alpha_{j}}^{j}\right)$ satisfy (a), (b), (c) as above; we allow permutations $\pi$ of the indices as in (d), provided the same permutation is used for $\left(\alpha_{1}\right),\left(\alpha_{1} \alpha_{2}\right), \ldots,\left(\alpha_{1}, \ldots, \alpha_{n}\right)$. Notice that, in view of (c) for each $\varepsilon>0$ there are only finitely many values of the multiindex $\left(\alpha_{1}, \ldots, \alpha_{n}\right)$ such that

$$
\min _{1 \leqq j \leqq n} \xi_{\alpha_{1} \ldots \alpha_{j}} \geqq \varepsilon .
$$

In view of this a cascade-considered as an unordered sequence of points in $\mathbb{R}_{>}^{n}$ - can be identified with an element of $\mathscr{X}\left(\mathbb{R}_{>}^{n}\right)$ as defined in Sect. 1 . Using the topology of $\mathscr{X}\left(\mathbb{R}_{>}^{n}\right)$ we obtain a Borel structure on the cascades, which will allow us to define measures.

To define the probability measure $\tilde{m}_{n}\left(d \tilde{X}^{(n)}\right)$ we first have to choose $n$ numbers $x_{j}$ between 0 and 1: $0<x_{1}<x_{2}<\cdots<x_{n}<1$. The definition will proceed in several steps.

First, we introduce a probability measure $v_{n}\left(d Y^{(n)}\right)$ on cascades $Y^{(n)}=$ $\left(\eta_{\alpha_{1}}^{1}, \ldots, \eta_{\alpha_{1} \ldots \alpha_{n}}^{n}\right)$. We proceed by induction. For $n=1$, we let $v_{1}\left(d Y^{(1)}\right)=m_{x_{1}}\left(d Y^{(1)}\right)$ as in Sect. 2. For $n>1$ we impose that

$$
\text { (image of } \left.v_{n}\left(d Y^{(n)}\right) \text { by the map } Y^{(n)} \rightarrow Y^{(n-1)}=\left(\eta_{\alpha_{1}}, \ldots, \eta_{\alpha_{1} \ldots \alpha_{n-1}}\right)\right)=v_{n-1}\left(d Y^{(n-1)}\right) \text {. }
$$

For fixed $\alpha_{1}, \ldots, \alpha_{n-1}$, let us write $Y_{\alpha_{1} \ldots \alpha_{n-1}}^{n}=\left(\eta_{\alpha_{1} \ldots \alpha_{n}}\right)$, the definition of $v_{n}\left(d Y^{(n)}\right)$ is completed by requiring that the $Y_{\alpha_{1} \ldots \alpha_{n-1}}^{n}$ have independent identical distributions given by $m_{x_{n}}(d Y)$.

As a second step we define a map $p: Y^{(n)} \rightarrow X^{(n)}$ of cascades such that

$$
\begin{aligned}
\left(\xi_{\alpha_{1}}^{1}, \xi_{\alpha_{1} \alpha_{2}}^{2}, \ldots, \xi_{\alpha_{1} \ldots \alpha_{n}}^{n}\right) & =p\left(\eta_{\alpha_{1}}^{1}, \eta_{\alpha_{1} \alpha_{2}}^{2}, \ldots, \eta_{\alpha_{1} \ldots \alpha_{n}}^{n}\right) \\
& =\left(\eta_{\alpha_{1}}^{1}, \eta_{\alpha_{1}}^{1} \eta_{\alpha_{1} \alpha_{2}}^{2}, \ldots, \prod_{j=1}^{n} \eta_{\alpha_{1} \ldots \alpha_{j}}^{j}\right)
\end{aligned}
$$

and let $m_{n}\left(d X^{(n)}\right)$ be the image of $v_{n}\left(d Y^{(n)}\right)$ by $p$. To summarize: the probability measure $m_{n}\left(d X^{(n)}\right)$ is obtained by picking first $X^{1}=\left(\xi_{\alpha_{1}}^{1}\right)$ according to the Poisson distribution $m_{x_{1}}\left(d X^{1}\right)$, then for each $\alpha_{1}$ picking the unordered sequence $Y_{\alpha_{1}}^{2}$ 
according to $m_{x_{2}}\left(d Y_{\alpha_{1}}^{2}\right)$ and writing $X_{\alpha_{1}}^{2}=\xi_{\alpha_{1}}^{1} Y_{\alpha_{1}}^{2}=\left(\xi_{\alpha_{1} \alpha_{2}}^{2}\right)$. Similarly, $X_{\alpha_{1} \alpha_{2}}^{3}=$ $\xi_{\alpha_{1} \alpha_{2}}^{2} Y_{\alpha_{1} \alpha_{2}}^{3}=\left(\xi_{\alpha_{1} \alpha_{2} \alpha_{3}}^{3}\right)$, etc. In this manner we obtain a probability distribution on cascades $X^{(n)}=\left(\xi_{\alpha_{1}}^{1}, \xi_{\alpha_{1} \alpha_{2}}^{2}, \xi_{\alpha_{1} \alpha_{2} \alpha_{3}}^{3} \cdots\right)$; this is precisely $m_{n}\left(d X^{(n)}\right)$.

For the third step we let $X^{(n)} \rightarrow \tilde{X}^{(n)}$ be the map defined by

$$
\left(\xi_{\alpha_{1}}^{1}, \xi_{\alpha_{1} \alpha_{2}}^{2}, \ldots, \xi_{\alpha_{1} \alpha_{2} \ldots \alpha_{n}}^{n}\right) \rightarrow\left(\widetilde{\xi}_{\alpha_{1}}^{1}, \widetilde{\xi}_{\alpha_{1} \alpha_{2}}^{2}, \ldots, \tilde{\xi}_{\alpha_{1} \alpha_{2} \ldots \alpha_{n}}^{n}\right)
$$

where

$$
\xi_{\alpha_{1} \alpha_{2} \ldots \alpha_{n}}^{n}=\xi_{\alpha_{1} \alpha_{2} \ldots \alpha_{n}}^{n} / \sum_{\beta_{1} \beta_{2} \ldots \beta_{n}} \xi_{\beta_{1} \beta_{2} \ldots \beta_{n}}^{n}
$$

and

$$
\tilde{\xi}_{\alpha_{1} \ldots \alpha_{j-1}}^{j-1}=\sum_{\beta_{j}} \tilde{\xi}_{\alpha_{1} \ldots \alpha_{j-1} \beta_{j}}^{j}
$$

Notice that $\tilde{X}^{(n)}$ is completely determined by the unordered family $\left(\xi_{\alpha_{1} \ldots \alpha_{n}}^{n}\right)$, i.e., by the last component of $X^{(n)}$. The image of $m_{n}\left(d X^{(n)}\right)$ by the map $X^{(n)} \rightarrow \widetilde{X}^{(n)}$ is the desired probability measure $\tilde{m}_{n}\left(d \tilde{X}^{(n)}\right)^{4}$.

For the formulation of the proposition below, it is convenient to write $m_{n}\left(d X^{(n)}\right)=m_{\left\{x_{1}, \ldots x_{n}\right\}}\left(d X^{(n)}\right)$ and similarly for $\tilde{m}_{n}\left(d \tilde{X}^{(n)}\right)$.

3.1. Proposition. Omitting $\tilde{\xi}_{j}$ from $\left(\tilde{\xi}_{1}, \ldots, \tilde{\xi}_{n}\right)$ defines a map $\tilde{X}^{(n)} \rightarrow \tilde{X}_{*}^{(n-1)}$. The image of $\tilde{m}_{\left\{x_{1}, \ldots, x_{n}\right\}}\left(d \tilde{X}^{(n)}\right)$ by this map is $\tilde{m}_{\left\{x_{1}, \ldots, x_{n}\right\} \backslash\left\{x_{j}\right\}}\left(d \tilde{X}_{*}^{(n-1)}\right)$.

We consider first the case $j<n$. For simplicity, we omit the indices $\alpha_{1}, \ldots, \alpha_{j-2}$, and write $\alpha_{j-1}=\alpha, \alpha_{j}=\beta, \alpha_{j+1}=\gamma$, so that

$$
X^{(n)}=\left(\ldots, \xi_{\alpha}^{j-1}, \xi_{\alpha}^{j-1} \eta_{\alpha \beta}^{j}, \xi_{\alpha}^{j-1} \eta_{\alpha \beta}^{j} \eta_{\alpha \beta \gamma}^{j+1}, \ldots\right),
$$

where $\left(\eta_{\alpha \beta}^{j}\right)$ is distributed-for fixed $\alpha$-according to $m_{x_{j}}(d Y)$, and similarly for $\eta_{\alpha \beta \gamma}^{j+1}$, etc. If $\xi_{\alpha}^{j-1}$ and $\eta_{\alpha \beta}^{j}$ are fixed, the distribution of $\left(\xi_{\alpha}^{j-1} \eta_{\alpha \beta}^{j} \eta_{\alpha \beta \gamma}^{j+1}\right)$ is Poisson, with density $\left(\xi_{\alpha}^{j-1} \eta_{\alpha \beta}^{j}\right)^{x_{j+1}} \varphi_{x_{j+1}}($.$) (see Proposition 2.2). Therefore, if \xi_{\alpha}^{j-1}$ and $\left(\xi_{\alpha \beta}^{j}\right)$ are fixed, the distribution of $\left(\xi_{\alpha}^{j-1} \eta_{\alpha \beta}^{j} \eta_{\alpha \beta \gamma}^{j+1}\right)$ is Poisson, with density

$$
\left(\xi_{\alpha}^{j-1}\right)^{x_{j+1}} \sum_{\beta}\left(\eta_{\alpha \beta}^{i}\right)^{x_{j+1}} \varphi_{x_{j+1}}(.)
$$

We write

$$
c_{\alpha}=\left(\sum_{\beta}\left(\eta_{\alpha \beta}^{j}\right)^{x_{j+1}}\right)^{1 / x_{j+1}}
$$

and

$$
X_{*}^{(n-1)}=\left(\ldots, \xi_{\alpha}^{j-1}, \xi_{\alpha}^{j-1} c_{\alpha} \eta_{* \alpha \delta}^{j+1}, \ldots\right)
$$

where $\left(\eta_{* \alpha \delta}^{j+1}\right)$ is distributed-for fixed $\alpha$-according to $m_{x_{j+1}}\left(d Y_{*}\right)$. For the

4 In the original language of Boltzmann factors we would have

$$
\xi_{\alpha_{1} \ldots \alpha_{n}}^{n}=\exp -\beta\left(\varepsilon_{\alpha_{1}}+\cdots+\varepsilon_{\alpha_{1} \ldots \alpha_{n}}\right),
$$

where the $\varepsilon_{\alpha_{1} \ldots \alpha_{j}}$ have a Poisson distribution with density $\rho_{j} e^{\rho_{j} \varepsilon}$, and $\rho_{j} / \beta=x_{j}$. The $\xi_{\alpha_{1} \ldots \alpha_{n}}^{n}$ are the normalized probabilities corresponding to these Boltzmann factors 
determination of $\tilde{X}_{*}^{(n-1)}$, it is equivalent to start from

or

$$
X_{*}^{(n-1)}=\left(\ldots, \xi^{j-2}, \xi^{j-2} \eta_{\alpha}^{j-1}, \xi^{j-2} \eta_{\alpha}^{j-1} c_{\alpha} \eta_{* \alpha \delta}^{j+1}, \ldots\right)
$$

$$
X_{* *}^{(n-1)}=\left(\ldots, \xi^{j-2}, \xi^{j-2} \eta_{\alpha}^{j-1} c_{\alpha}, \xi^{j-2} \eta_{\alpha}^{j-1} c_{\alpha} \eta_{* \alpha \delta}^{j+1}, \ldots\right)
$$

because the last components are the same. We may also write

$$
X_{* *}^{(n-1)}=\left(\ldots, \xi^{j-2}, \xi^{j-2} \eta_{\alpha}^{*}, \xi^{j-2} \eta_{\alpha}^{*} \eta_{* \alpha \delta}^{j+1}, \ldots\right)
$$

where $\eta_{\alpha}^{*}=\eta_{\alpha}^{j-1} c_{\alpha}$. We shall now need the following:

3.2. Lemma. $\left(\eta_{\alpha}^{*}\right)$ has Poisson distribution with density $C \varphi_{x_{i-1}}\left(\eta^{*}\right)$ for some constant $C>0$.

The $c_{\alpha}$ are independently identically distributed with some density $h$ and, approximating $\varphi_{x_{j-1}}$ by an integrable function, one sees that $\left(\eta_{\alpha}^{*}\right)$ has Poisson distribution with density $\varphi$ such that

$$
\begin{aligned}
\varphi(\eta) & =\int_{0}^{\infty} h(u) d u \int_{0}^{\infty} \varphi_{x_{j-1}}(v) d v \delta(\eta-u v)=\int_{0}^{\infty} h(u) d u \int_{0} \varphi_{x_{j-1}}(v) d v \frac{1}{u} \delta(v-\eta / u) \\
& =\int_{0}^{\infty} h(u) \frac{d u}{u} \varphi_{x_{j-1}}(\eta / u)=\varphi_{x_{j-1}}(\eta) \int_{0}^{\infty} h(u) u^{x_{j-1}+1} \frac{d u}{u} \\
& =\varphi_{x_{j-1}}(\eta) \int_{0}^{\infty} h(u) u^{x_{j-1}} d u=C \varphi_{x_{j-1}}(\eta),
\end{aligned}
$$

provided the last integral above converges. According to (3.1) this integral is the expectation value of

$$
\left(\sum_{\beta}\left(\eta_{\alpha \beta}^{j}\right)^{x_{j+1}}\right)^{x_{j-1} / x_{j+1}}
$$

and is easily seen to be finite; in fact

$$
C=\int m_{x_{j}}(d Y)\left(\sum_{\beta}\left(\eta_{\beta}\right)^{x_{j+1}}\right)^{x_{j-1} / x_{j+1}}=\int m_{x_{j} / x_{j+1}}\left(d Y^{*}\right)\left|Y^{*}\right|^{x_{j-1} / x_{j+1}}=\int m_{x}(d Y)|Y|^{s},
$$

with $x=x_{j} / x_{j+1}, s=x_{j-1} / x_{j+1}$.

We return now to the proof of Proposition 3.1. In view of the above lemma we may write $\eta_{\alpha}^{*}=C^{*} \eta_{\alpha}^{* *}$, where $\left(\eta_{\alpha}^{* *}\right)$ has Poisson distribution with density $\varphi_{x_{j-1}}\left(\eta^{* *}\right)$ provided $C^{* x_{j-1}}=C$, and $C$ is given by (3.2). We do not change $\tilde{X}_{*}^{(n-1)}$ if we replace $\eta_{\alpha}^{*}$ by $\eta_{\alpha}^{* *}$ in $X_{* *}^{(n-1)}$, so that we may obtain $\tilde{X}_{*}^{(n-1)}$ from

$$
X_{* * *}^{(n-1)}=\left(\ldots, \xi^{j-2}, \xi^{j-2} \eta_{\alpha}^{* *}, \xi^{j-2} \eta_{\alpha}^{* *} \eta_{* \alpha \delta}^{j+1}, \ldots\right)
$$

where $\left(\eta_{\alpha}^{* *}\right)$ and $\left(\eta_{* \alpha \delta}^{j+1}\right)$ have Poisson distributions with densities $\varphi_{x_{j-1}}$ and $\varphi_{x_{j+1}}$ respectively. Therefore $\tilde{X}_{*}^{(n-1)}$ is distributed according to $\tilde{m}_{\left\{x_{1}, \ldots, x_{n}\right\} \backslash\left\{x_{j}\right\}}\left(d \tilde{X}_{*}^{(n-1)}\right)$, proving the Proposition for $j<n$.

For $j=n$, we have

$$
X^{(n)}=\left(\ldots, \xi^{n-2}, \xi^{n-2} \eta_{\alpha}^{n-1}, \xi^{n-2} \eta_{\alpha}^{n-1} \eta_{\alpha \beta}^{n}\right),
$$

where $\left(\eta_{\alpha \beta}^{n}\right)$ is distributed-for fixed $\alpha$-according to $m_{x_{n}}(d Y)$. We can determine 
$\tilde{X}_{*}^{(n-1)}$ from

$$
X_{*}^{(n-1)}=\left(\ldots, \xi^{n-2}, \xi^{n-2} \xi_{\alpha}^{n-1} \sum_{\beta} \eta_{\alpha \beta}^{n}\right)
$$

which has the appropriate last component. Writing $c_{\alpha}=\sum_{\beta} \eta_{\alpha \beta}^{n}, \eta_{\alpha}^{*}=\eta_{\alpha}^{n-1} c_{\alpha}$, we can proceed as in the case $j<n$, with the same conclusion.

3.3. Corollary. The map $\tilde{X}^{(n)} \rightarrow \tilde{X}^{j}$ defined by $\left(\tilde{\xi}_{\alpha_{1}}^{1}, \ldots, \tilde{\xi}_{\alpha_{1} \ldots \alpha_{n}}^{n}\right) \rightarrow\left(\tilde{\xi}_{\alpha_{1} \ldots \alpha_{j}}^{j}\right)$ sends $\tilde{m}_{\left\{x_{1}, \ldots, x_{n}\right\}}\left(d \tilde{X}^{(n)}\right)$ to $\tilde{m}_{x_{j}}\left(d \tilde{X}^{j}\right)$.

\section{Continuous Probability Cascades}

Using an interval $I \subset(0,1)$ instead of the finite set $\left\{x_{1}, \ldots, x_{n}\right\}$ we shall replace the $\tilde{X}^{(n)}$ by continuous probability cascades $Z$, and the probability measure $\tilde{m}_{n}\left(d \tilde{X}^{(n)}\right)$ by $\tilde{m}(d Z)$.

Remember that we have

$$
\tilde{X}^{(n)}=\left(\widetilde{\xi}_{\alpha_{1}}^{1}, \tilde{\xi}_{\alpha_{1} \alpha_{2}}^{2}, \ldots, \tilde{\xi}_{\alpha_{1} \ldots \alpha_{n}}^{n}\right)
$$

with $\alpha_{1}, \ldots, \alpha_{n}$ belonging to countable infinite index sets, such that

$$
\begin{aligned}
& \xi_{\alpha_{1} \ldots \alpha_{j}}^{j}>0, \\
& \sum_{\alpha_{j}} \xi_{\alpha_{1} \ldots \alpha_{j}}^{j}=\left\{\begin{array}{ll}
\xi_{\alpha_{1} \ldots \alpha_{j-1}}^{j-1} & \text { for } j>1 \\
1 & \text { for } j=1
\end{array},\right.
\end{aligned}
$$

and permutations $\pi$ preserving the tree structure of $\left\{\alpha_{1}, \ldots, \alpha_{n}\right)$ are allowed.

We shall now introduce more general cascades $Z^{(n)}$ to form a set $K_{\left\{x_{1}, \ldots, x_{n}\right\}}$ with compact topology. We write

$$
Z^{(n)}=\left(z_{\alpha_{1}}, z_{\alpha_{1} \alpha_{2}}, \ldots, z_{\alpha_{1} \ldots \alpha_{n}}\right)
$$

with the following restrictions:

(a) $z_{\alpha_{1} \ldots \alpha_{j}}>0$

(b) Given $\alpha_{1}, \ldots, \alpha_{j-1}$, the index $\alpha_{j}$ varies in $\alpha$ countable set, which may be infinite or finite

$$
\text { (c) } \sum_{\alpha_{j}} z_{\alpha_{1} \ldots \alpha_{j}} \leqq \begin{cases}z_{\alpha_{1} \cdots \alpha_{j-1}} & \text { for } j>1 \\ 1 & \text { for } j=1\end{cases}
$$

(d) We allow permutations $\pi$ : $\left(\alpha_{1}, \ldots, \alpha_{n}\right) \rightarrow\left(\alpha_{1}^{\prime}, \ldots, \alpha_{n}^{\prime}\right)$ such that $\left(\alpha_{1}=\beta_{1}, \ldots, \alpha_{j-1}=\beta_{j-1}, \alpha_{j} \neq \beta_{j}\right)$ implies $\left(\alpha_{1}^{\prime}=\beta_{1}^{\prime}, \ldots, \alpha_{j-1}^{\prime}=\beta_{j-1}^{\prime}, \alpha_{j}^{\prime} \neq \beta_{j}^{\prime}\right)$.

Given $\varepsilon>0$ and a finite set $S$ of multiindices $\left(\alpha_{1}, \ldots, \alpha_{n}\right)$, a neighborhood $u$ of $Z^{(n)}$ in $K_{\left\{x_{1}, \ldots, x_{n}\right\}}$ is defined by the condition that for each $\left(\alpha_{1}, \ldots, \alpha_{n}\right) \in S$ there be $\left(\alpha_{1}^{\prime}, \ldots, \alpha_{n}^{\prime}\right)$ such that

$$
\max _{1 \leqq j \leqq n}\left|z_{\alpha_{1}^{\prime} \cdots \alpha_{j}^{\prime}}^{\prime}-z_{\alpha_{1} \cdots \alpha_{j}}\right|<\varepsilon .
$$

It is not hard to see that $K_{\left\{x_{1}, \ldots, x_{n}\right\}}$ is metrizable compact with this topology. [Note that, for every $\varepsilon>0$, there is $N(\varepsilon)$ such that, for all $Z^{(n)} \in K_{\left\{x_{1}, \ldots, x_{n}\right\}}$, there are at most 
$N(\varepsilon)$ values of the multiindex $\left(\alpha_{1}, \ldots, \alpha_{n}\right)$ such that $\left.\min z_{\alpha_{1} \ldots \alpha_{j}} \geqq \varepsilon\right]$.

If $\left\{x_{1}, \ldots, x_{n}\right\} \subset\left\{x_{1}^{\prime}, \ldots, x_{N}^{\prime}\right\}$, there is a natural map

$$
K_{\left\{x_{1}^{\prime}, \ldots, x_{N}^{\prime}\right\}} \rightarrow K_{\left\{x_{1}, \ldots, x_{n}\right\}},
$$

obtained by deleting from $Z^{\prime(N)}$ the $\left(z_{\alpha_{1}^{\prime} \cdots \alpha_{j}^{\prime}}^{\prime}\right)$ such that $x_{j}^{\prime} \notin\left\{x_{1}, \ldots, x_{n}\right\}$, and lumping together indices as needed. This map is clearly continuous and onto. Under the above conditions, a natural construction ("inverse limit" or "projective limit") gives a compact set $K$, and continuous maps of $K$ onto all $K_{\left\{x_{1}, \ldots, x_{n}\right\}}$ commuting with the maps of (4.1). Because of the compatibility conditions of Proposition 3.1 , there is a natural measure $\tilde{m}(d Z)$ on $K$ which is mapped to $\tilde{m}_{\left\{x_{1}, \ldots, x_{n}\right\}}\left(d X^{(n)}\right)$ on each $K_{\left\{x_{1}, \ldots, x_{n}\right\}} ; \tilde{m}(d Z)$ is the projective limit of the $\tilde{m}_{\left\{x_{1}, \ldots, x_{n}\right\}}\left(d X^{(n)}\right)$ [see N. Bourbaki [1] Integration, Ch. III, §4, $n^{\circ} 5$ ].

We have thus completed the promised continuous extension of Derrida's GREM. A word of caution is however in order. The compact set $K$ is not metrizable, which means that we do not have the standard setup of integration theory, and some theorems do not apply. We would obtain a metrizable set by taking the projective limit of the $K_{\left\{x_{1}, \ldots, x_{n}\right\}}$, where the $x_{i}$ are restricted to the set of rationals (which is countable); this definition has however the disadvantage of some arbitrariness. The two definitions are however compatible, and it is not inconvenient in practice to use both, with the advantage that one is canonical and the other leads to a standard probability measure.

We conclude this section by an example of calculation, namely that of the correlation functions $\rho_{x_{1} \ldots x_{n}}\left(p_{1}, \ldots, p_{n}\right)$ of a cascade. By definition, $\rho_{x_{1}} \cdots x_{n}\left(p_{1}, \ldots, p_{n}\right)$ is the probability density of finding a decreasing sequence of clusters of sizes $p_{1}>\cdots>p_{n}$ for the values $x_{1}, \ldots, x_{n}$ of the parameter $x$. Thus

$$
\rho_{x_{1} \ldots x_{n}}\left(p_{1}, \ldots, p_{n}\right)=\int \tilde{m}_{\left\{x_{1}, \ldots, x_{n}\right\}}\left(d \tilde{X}^{(n)}\right) \sum_{\alpha_{1} \ldots \alpha_{n}} \delta\left(p_{1}-\tilde{\xi}_{\alpha_{1}}^{1}\right) \cdots \delta\left(p_{n}-\tilde{\xi}_{\alpha_{1} \ldots \alpha_{n}}^{n}\right) .
$$

4.1. Proposition. The correlation functions are given by

$$
\rho_{x_{1} \ldots x_{n}}\left(p_{1}, \ldots, p_{n}\right)=\frac{\left(1-p_{1}\right)^{x_{1}-1}}{\Gamma\left(x_{1}\right)} \frac{\left(p_{1}-p_{2}\right)^{x_{2}-x_{1}-1}}{\Gamma\left(x_{2}-x_{1}\right)} \cdots \frac{\left(p_{n-1}-p_{n}\right)^{x_{n}-x_{n-1}-1}}{\Gamma\left(x_{n}-x_{n-1}\right)} \cdot \frac{p_{n}^{-x_{n}-1}}{\Gamma\left(1-x_{n}\right)} .
$$

Let us write

$$
\begin{aligned}
& \mathscr{V}=\sum_{\beta_{1} \ldots \beta_{n}} \eta_{\beta_{1}} \eta_{\beta_{1} \beta_{2}} \cdots \eta_{\beta_{1} \ldots \beta_{n}}=\left|X^{n}\right| \\
& \mathscr{V}_{\alpha_{1} \ldots \alpha_{j}}=\sum_{\beta_{j+1} \cdots \beta_{n}} \eta_{\alpha_{1} \cdots \alpha_{j} \beta_{j+1}} \cdots \eta_{\alpha_{1} \ldots \beta_{n}} .
\end{aligned}
$$

We have then

$$
\begin{array}{r}
\rho_{x_{1} \ldots x_{n}}\left(p_{1}, \ldots, p_{n}\right)=m_{\left\{x_{1}, \ldots, x_{n}\right\}}\left(d X^{(n)}\right) \sum_{\alpha_{1}} \delta\left(p_{1}-\frac{\eta_{\alpha_{1}} \mathscr{V}_{\alpha_{1}}}{\mathscr{V}}\right) \\
\cdot \sum_{\alpha_{2}} \delta\left(p_{2}-p_{1} \frac{\eta_{\alpha_{1} \alpha_{2}} \mathscr{V}_{\alpha_{1} \alpha_{2}}}{\mathscr{V}_{\alpha_{1}}}\right) \cdots \sum_{\alpha_{n}} \delta\left(p_{n}-p_{n-1} \frac{\eta_{\alpha_{1} \ldots \alpha_{n}}}{\mathscr{V}_{\alpha_{1} \cdots \alpha_{n-1}}}\right)
\end{array}
$$




$$
\begin{aligned}
= & \int m_{\left\{x_{1}, \ldots, x_{n}\right\}}\left(d X^{(n)}\right) \mathscr{V} \sum_{\alpha_{1}}\left(p_{1} \frac{\mathscr{V}}{\mathscr{V}_{\alpha_{1}}}-\eta_{\alpha_{1}}\right) \sum_{\alpha_{2}} \delta\left(p_{2} \frac{\mathscr{V}_{\alpha_{1}}}{\mathscr{V}_{\alpha_{1} \alpha_{2}}}-p_{1} \eta_{\alpha_{1} \alpha_{2}}\right) \\
& \cdots \sum_{\alpha_{n}} \delta\left(p_{n} \mathscr{V}_{\alpha_{1} \cdots \alpha_{n-1}}-p_{n-1} \eta_{\alpha_{1} \ldots \alpha_{n}}\right) .
\end{aligned}
$$

We use now the same trick as for the calculation of $\rho_{x}(p)$ in Proposition 2.1, writing $\eta_{\alpha_{1}}=\eta_{1}, \ldots, \eta_{\alpha_{1} \ldots \alpha_{n}}=\eta_{n}$ and lumping the other variables as $V=\left(V_{1}, V_{2}, \ldots\right)$. The integration $d V$ will not be done explicitly.

We obtain thus

$$
\begin{aligned}
& \rho_{x_{1} \ldots x_{n}}\left(p_{1}, \ldots, p_{n}\right)=\int d V \int \varphi_{x_{1}}\left(\eta_{1}\right) d \eta_{1} \cdots \int \varphi_{x_{n}}\left(\eta_{n}\right) d \eta_{n}\left(V_{1}+\eta_{1}\left(V_{2}+\eta_{2}\left(V_{3}+\cdots\right)\right)\right) \\
& \cdot \delta\left(p_{1} \frac{V_{1}}{V_{2}+\eta_{2}\left(V_{3}+\cdots\right)}-\left(1-p_{1}\right) \eta_{1}\right) \delta\left(p_{2} \frac{V_{2}}{V_{3}+\eta_{3}\left(V_{4}+\cdots\right)}-\left(p_{1}-p_{2}\right) \eta_{2}\right) \cdots \\
& \cdot \delta\left(p_{n-1} \frac{V_{n-1}}{V_{n}+\eta_{n}}-\left(p_{n-2}-p_{n-1}\right) \eta_{n-1}\right) \delta\left(p_{n} V_{n}-\left(p_{n-1}-p_{n}\right) \eta_{n}\right) .
\end{aligned}
$$

The $\delta$ 's in the integrand imply the following equalities:

$$
\begin{aligned}
\eta_{n} & =\frac{p_{n}}{p_{n-1}-p_{n}} V_{n}, & & V_{n}+\eta_{n}=\frac{p_{n-1}}{p_{n-1}-p_{n}} V_{n}, \\
\eta_{n-1} & =\frac{p_{n-1}-p_{n}}{p_{n-2}-p_{n-1}} \cdot \frac{V_{n-1}}{V_{n}}, & & V_{n-1}+\eta_{n-1}\left(V_{n}+\eta_{n}\right)=\frac{p_{n-2}}{p_{n-2}-p_{n-1}} V_{n-1}, \\
& \ldots & & p_{2}-p_{3} \\
\eta_{2} & =\frac{V_{2}}{p_{1}-p_{2}}, & & V_{2}+\eta_{2}\left(V_{3}+\cdots\right)=\frac{p_{1}}{p_{1}-p_{2}} V_{2}, \\
\eta_{1} & =\frac{p_{1}-p_{2}}{1-p_{1}} \cdot \frac{V_{1}}{V_{2}}, & & V_{1}+\eta_{1}\left(V_{2}+\eta_{2}\left(V_{3}+\cdots\right)\right)=\frac{1}{1-p_{1}} V_{1} .
\end{aligned}
$$

We have thus

$$
\begin{aligned}
& \rho_{x_{1} \ldots x_{n}}\left(p_{1}, \ldots, p_{n}\right)=\left(\frac{p_{1}-p_{2}}{1-p_{1}}\right)^{-x_{1}-1}\left(\frac{p_{2}-p_{3}}{p_{1}-p_{2}}\right)^{-x_{2}-1} \cdots\left(\frac{p_{n-1}-p_{n}}{p_{n-2}-p_{n-1}}\right)^{-x_{n-1}-1} \\
& \cdot\left(\frac{p_{n}}{p_{n-1}-p_{n}}\right)^{-x_{n}-1} \cdot \frac{1}{1-p_{1}} \cdot \frac{1}{1-p_{1}} \cdot \frac{1}{p_{1}-p_{2}} \cdots \frac{1}{p_{n-2}-p_{n-1}} \cdot \frac{1}{p_{n-1}-p_{n}} \\
& \cdot \int d V V_{1} \varphi_{x_{1}}\left(\frac{V_{1}}{V_{2}}\right) \cdots \varphi_{x_{n-1}}\left(\frac{-V_{n-1}}{V_{n}}\right) \varphi_{x_{n}}\left(V_{n}\right) \\
= & K_{n}\left(1-p_{1}\right)^{x_{1}-1}\left(p_{1}-p_{2}\right)^{x_{2}-x_{1}-1} \cdots\left(p_{n-1}-p_{n}\right)^{x_{n}-x_{n-1}-1} p_{n}^{-x_{n}-1},
\end{aligned}
$$

where

$$
K_{n}=\int d V V_{1} \varphi_{x_{1}}\left(\frac{V_{1}}{V_{2}}\right) \cdots \varphi_{x_{n-1}}\left(\frac{V_{n-1}}{V_{n}}\right) \varphi_{x_{n}}\left(V_{n}\right)
$$


We know that

$$
K_{1}=\frac{1}{\Gamma\left(x_{1}\right) \Gamma\left(1-x_{1}\right)}
$$

On the other hand

$$
\int \rho_{x_{1} \ldots x_{n}}\left(p_{1}, \ldots, p_{n}\right) p_{n} d p_{n}=\rho_{x_{1} \cdots x_{n-1}}\left(p_{1}, \ldots, p_{n-1}\right) p_{n-1},
$$

so that

$$
\begin{aligned}
K_{n-1} & =p_{n-1}^{x_{n-1}} K_{n} \int\left(p_{n-1}-p_{n}\right)^{x_{n}-x_{n-1}-1} p_{n}^{-x_{n}} d p_{n} \\
& =K_{n} \int(1-p)^{x_{n}-x_{n-1}-1} p^{-x_{n}} d p=K_{n} \frac{\Gamma\left(1-x_{n}\right) \Gamma\left(x_{n}-x_{n-1}\right)}{\Gamma\left(1-x_{n-1}\right)} .
\end{aligned}
$$

By induction, we see therefore that the coefficient given in (4.2) is correct.

4.2. Remark. Applying $\int d p_{j}$, with $j<n$, to $\rho_{x_{1} \ldots x_{n}}\left(p_{1}, \ldots, p_{n}\right)$ yields the correlation function of $n-1$ arguments corresponding to the set $\left\{x_{1}, \ldots, x_{n}\right\} \backslash\left\{x_{j}\right\}$ of values of $x$. [This is readily checked from (4.2)]. Applying $\int d p_{n}$ yields $\infty$. Applying $\int p_{n} d p_{n}$ yields $\rho_{x_{1} \cdots x_{n-1}}\left(p_{1}, \ldots, p_{n-1}\right) \cdot p_{n-1}$ as already noted.

4.3. Remark. It is to be expected that changes in the temperature scale will have a simple effect on the measures $\tilde{m}_{x}(d X), \tilde{m}_{n}\left(d X^{(n)}\right), \tilde{m}(d Z)$. The appropriate results are stated and proved in Appendix 2.

\section{Conclusions}

In this note we have shown that the REM and the GREM are "universal" probability structures depending on very few parameters: $x$ for the REM, and the interval I for the GREM. It is not too astonishing that these structures should occur in the study of disordered systems. Note that, for the SK model, random free energies rather than random energies are involved; in this case we should take $I=(0, x)$ with some $x$ depending on the temperature. Of course, the relevance of REM and GREM to the SK model depend on Parisi's solution being correct - which is not proved. On the other hand the treatment of the SK model based on Parisi's Ansatz yields more specific results than those captured in the REM and GREM. It is nevertheless interesting that a number of the explicit calculations pertaining to Parisi's solution only depend on the REM and GREM. One can therefore-as we have shown-perform these calculations more systematically than is usually done in the literature.

\section{Appendix 1. The Space $\mathscr{X}(\mathcal{O})$}

Let $\mathcal{O}$ be a locally compact (Hausdorff) topological space. We shall assume for simplicity that the topology of $\mathcal{O}$ has a countable basis. We denote by $\mathscr{X}(\mathcal{O})$ the space of functions $X: \mathcal{O} \mapsto \mathbb{N}$ such that

$$
\sum_{\xi \in K} X(\xi)<+\infty \text { for every compact set } K \subset \mathcal{O} .
$$


Clearly, $\mathscr{X}(\mathcal{O})$ is the disjoint union $\cup_{m \in \mathbb{N}} \mathscr{X}^{m} \cup \mathscr{X}^{\infty}$, where

$$
\mathscr{X}^{m}=\left\{X \in \mathscr{X}(\mathcal{O}): \sum_{\xi \in \mathcal{O}} X(\xi)=m\right\},
$$

and we may identify the elements of $\mathscr{X}^{m}, m$ finite or $\infty$, with unordered sequences $\left(\xi_{\alpha}\right)$, where the index set has $m$ elements, and permutations of the index set are allowed.

We define a topology on $\mathscr{X}(\mathcal{O})$ by giving a basis of neighborhoods $\left\{\mathscr{N}_{X}\left(\psi_{1}, \ldots, \psi_{k}\right)\right\}$ of $X$. If $\psi_{1}, \ldots, \psi_{k}$ are real continuous functions with compact support in $\mathcal{O}$, we define

$$
\mathscr{N}_{X}\left(\psi_{1}, \ldots, \psi_{k}\right)=\left\{Y \in \mathscr{X}(\mathcal{O}):\left|\sum_{\xi \in \mathcal{O}} \psi_{i}(\xi)(X(\xi)-Y(\xi))\right|<1 \text { for } i=1, \ldots, k\right\} .
$$

It is easily seen that this topology is compatible with a metric for which $\mathscr{X}(\mathcal{O})$ is complete $^{5}$. Let us associate with $X \in \mathscr{X}(\mathcal{O})$ the measure on $\mathcal{O}$ defined by

$$
\delta_{X}=\sum_{\xi \in \mathcal{O}} X(\xi) \delta_{\xi},
$$

where $\delta_{\xi}$ is the unit mass at $\xi$. Then, $X \mapsto \delta_{X}$ is a homeomorphism of $\mathscr{X}(\mathcal{O})$ to $\left\{\delta_{X}: X \in \mathscr{X}(\mathcal{O})\right\}$ equipped with the vague topology of measures.

Proposition. The Borel subsets of $\mathscr{X}(\mathcal{O})$ constitute the $\sigma$-ring generated by the sets

$$
\mathscr{W}_{B}^{m}=\left\{X \in \mathscr{X}(\mathcal{O}): \sum_{\xi \in B} X(\xi)=m\right\}
$$

where $m \in \mathbb{N}$ and $B$ is a relatively compact Borel subset of $\mathcal{O}$.

The proof is easy, and left to the reader.

\section{Appendix 2. Changes of Temperature Scale}

Dividing the temperature by a number $\lambda>0$ has the effect of replacing the Boltzmann factor $\xi=e^{-\beta E}$ by $\xi^{\lambda}=e^{-\lambda \beta E}$. The map $f: \mathbb{R}_{>} \mapsto \mathbb{R}_{>}$defined by $f \xi=\xi^{\lambda}$ sends the Poisson distribution $m_{x}$ with density $\varphi_{x}$ to a Poisson distribution $m_{\varphi^{*}}$, where $\varphi^{*}$ is determined by (1.4):

or

$$
\varphi^{*}(\eta)=\left|\lambda \xi^{\lambda-1}\right|^{-1} \varphi_{x}(\xi), \quad \text { with } \eta=\xi^{\lambda}
$$

$$
\varphi^{*}(\eta)=\frac{x \xi^{-x-1}}{\lambda \xi^{\lambda-1}}=\frac{x}{\lambda} \xi^{-x-\lambda}=\frac{x}{\lambda} \eta^{-x / \lambda-1}=\varphi_{x / \lambda}(\eta) .
$$

A.1. Proposition. Let $\lambda>x$.

(a) The map $X=\left(\xi_{\alpha}\right) \mapsto X^{\lambda}=\left(\left(\xi_{\alpha}\right)^{\lambda}\right)$ sends $m_{x}$ to $m_{x / \lambda}$.

(b) If $\tilde{X}=\left(\tilde{\xi}_{\alpha}\right)$, write $\tilde{\eta}_{\alpha}=\left(\tilde{\xi}_{\alpha}\right)^{\lambda} / \sum_{\beta}\left(\tilde{\xi}_{\beta}\right)^{\lambda}$. Then, the map $\tilde{X} \rightarrow \tilde{X}^{\lambda} /\left|\tilde{X}^{\lambda}\right|=\left(\tilde{\eta}_{\alpha}\right)$ sends $\tilde{m}_{x}$ to $\tilde{m}_{x / \lambda}$.

$5 \mathrm{I}$ am indebted to $\mathrm{O}$. Lanford for pointing this out to me a number of years ago. Notice that the existence of the metric requires the topology of $\mathcal{O}$ to have a countable basis 
(a) has been proved above; (b) results from the commutative diagram of maps:

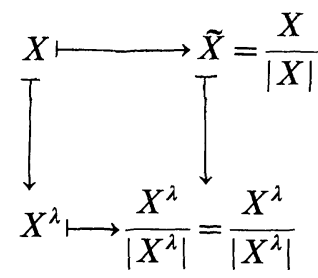

A.2. Proposition. Let $0<x_{1}<x_{2}<\cdots<x_{n}<1$, and $\lambda>x_{n}$.

(a) The map $X^{(n)}=\left(\xi_{\alpha_{1}}^{1}, \xi_{\alpha_{1} \alpha_{2}}^{2}, \ldots, \xi_{\alpha_{1} \ldots \alpha_{n}}^{n}\right) \mapsto X^{(n) \lambda}=\left(\left(\xi_{\alpha_{1}}^{1}\right)^{\lambda},\left(\xi_{\alpha_{1} \alpha_{2}}^{2}\right)^{\lambda}, \ldots,\left(\xi_{\alpha_{1} \ldots \alpha_{n}}^{n}\right)^{\lambda}\right)$ sends the measure $m_{\left\{x_{1}, \ldots, x_{n}\right\}}$ to $m_{\left\{x_{1} / \lambda, \ldots, x_{n} / \lambda\right\}}$.

(b) If $\tilde{X}^{(n)}=\left(\tilde{\xi}_{\alpha_{1}}^{1}, \tilde{\xi}_{\alpha_{1} \alpha_{2}}^{2}, \ldots, \tilde{\xi}_{\alpha_{1} \ldots \alpha_{n}}^{n}\right)$, write

$$
\tilde{\eta}_{\alpha_{1} \ldots \alpha_{j}}^{j}=\sum_{\beta_{j+1}, \ldots, \beta_{n}}\left(\tilde{\xi}_{\alpha_{1} \ldots \alpha_{j}}^{n} \beta_{j+1} \ldots \beta_{n}\right)^{n} / \sum_{\beta_{1} \ldots \beta_{n}}\left(\tilde{\xi}_{\beta_{1} \ldots \beta_{n}}^{n}\right) .
$$

Then, the map $\tilde{X}^{(n)} \mapsto\left(\tilde{X}^{(n) \lambda}\right)^{\sim}=\left(\tilde{\eta}_{\alpha_{1}}^{1}, \tilde{\eta}_{\alpha_{1} \alpha_{2}}^{2}, \ldots, \tilde{\eta}_{\alpha_{1} \ldots \alpha_{n}}^{n}\right)$ sends the measure $\tilde{m}_{\left\{x_{1}, \ldots, x_{n}\right\}}$ to $\tilde{m}_{\left\{x_{1} /, \ldots, x_{n} / \lambda\right\}}$.

The proof of (a) is obtained by induction on $n$, using Proposition A.1 (a). From (a) and the commutative diagram

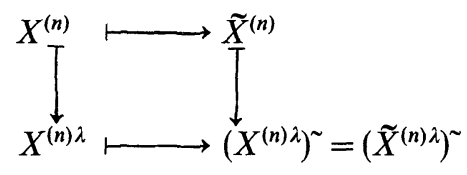

one gets (b).

Clearly, Proposition A.2 (b) can be adapted to continuous probability cascades: the measure $\tilde{m}(d Z)$ corresponding to the interval $I \subset(0,1)$ is sent to the measure corresponding to $I / \lambda$ (for instance, to $(0, x / \lambda]$ if $I=(0, x]$ and $\lambda>x$ ).

Acknowledgements. $\mathbf{I}$ am indebted to N. Sourlas, B. Derrida and C. de Dominicis for much needed guidance in the mystifying field of Parisi's Ansatz, REM and GREM.

\section{References}

1. Bourbaki, N.: Elements de mathématique, Livre VI. Intégration, chapitres 1, 2, 3 et 4, A.S.I. 1175, Paris: Hermann 1965

2. Derrida, B.: Random-energy model: Limit of a family of disordered models. Phys. Rev. Lett. 45, 79$82(1980)$

3. Derrida, B.: Random-energy model: An exactly solvable model of disordered systems. Phys. Rev. B24, 2613-2626 (1981)

4. Derrida, B.: A generalization of the random energy model which includes correlations between energies. J. Phys. Lett. 46, L-401-L-407 (1985)

5. Derrida, B., Gardner, E.: Solution of the generalized random energy model, J. Phys. C. In press (1986)

6. Derrida, B., Toulouse, G.: Sample to sample fluctuations in the random energy model. J. Phys. Lett. 46, L-223-L228 (1985)

7. de Dominicis, C., Hilhorst, H.: Random (free) energies in spin glasses, J. Phys. Lett. 46, L-909-L-914 (1985) 
8. Kirkpatrick, S., Sherrington, D.: Infinite-ranged models of spin-glasses, Phys. Rev. B17, 4384-4403 (1978)

9. Mézard, M., Parisi, G., Sourlas, N., Toulouse, G., Virasoro, M.: Replica symmetry breaking and the nature of the spin glass phase. J. Phys. 45, 843-854 (1984)

10. Mézard, M., Parisi, G., Virasoro, M.: Random free energies in spin glasses. J. Phys. Lett. 46, L-217-L222 (1985)

11. Mézard, M., Parisi, G., Virasoro, M.: SK model: The replica solution without replicas, Europhys. Lett. 1, 77-82 (1986)

12. Parisi, G.: Infinite number of order parameters for spin-glasses. Phys. Rev. Lett. 43, 1754-1756(1979)

13. Parisi, G.: Order parameter for spin-glasses. Phys. Rev. Lett. 50, 1946-1948 (1983)

14. Sherrington, D., Kirkpatrick, S.: Solvable model of a spin-glass. Phys. Rev. Lett. 35, 1792-1796(1975)

Communicated by T. Spencer

Received June 15, 1986 
